\title{
Exploration and application of natural and infrared color images in middle school
}

\section{Steven Croft, Stephen Pompea, Steven McGee}

Steven K. Croft, Stephen M. Pompea, Steven McGee, "Exploration and application of natural and infrared color images in middle school," Proc. SPIE 9663, Eighth International Topical Meeting on Education and Training in Optics and Photonics, 96631I (6 October 2003); doi: 10.1117/12.2208467

SPIE Event: Eighth International Topical Meeting on Education and Training in Optics and Photonics, 2003, Tucson, Arizona, United States 


\title{
Exploration and application of natural and infrared color images in middle school
}

\author{
Steven K. Croft, Stephen M. Pompea \\ National Optical Astronomy Observatory, Tucson, AZ \\ 520-318-8495; scroft@noao.edu \\ Steven McGee \\ Center for Educational Technologies, Wheeling, WV
}

\begin{abstract}
A new classroom-tested software package provides an innovative curriculum for teaching elementary concepts in spectrum and color. Students explore the construction of natural and infrared color composites of Earth scenes to determine the unique spectral characteristics of terrestrial plants and apply this knowledge to a search for life on Mars.

(C)2003 Optical Society of America
\end{abstract}

OCIS codes: (000.2060) Education; (100.2000) Digital image processing

\section{Introduction}

Natural and false color images are becoming increasingly common in displaying and explaining features and processes in the environment of Earth and the other planets. School books, magazines, and television programs rely on natural and false color images of clouds, biota, lakes and oceans, and bare rock to help students and public alike to understand important elements of the world around them: weather, floods, forest and crop management, mining and land management, etc., not to mention the use of such images to explore the other planets and the universe beyond. Understanding and interpreting these images requires understanding of basic concepts about the visible and invisible portions of the electromagnetic spectrum, the nature of color as perceived by the human eye, and the construction and use of such images. In a new software package "Astronomy Village: Investigating the Solar System (AVISS)," we provide a classroom-tested curriculum for teaching a basic introduction to these concepts in middle school.

\section{Description of AVISS Curriculum}

AVISS is a software package provided on two CD-ROMs, one CD providing teacher guidance and support, and the other containing the student materials. Students are provided with a simulated environment - a science community based on major observatories like Kitt Peak National Observatory - that includes library, research, and observing facilities and resources. Each facility contains imbedded materials and activities that the students use to investigate a particular question. Students are presented with ten possible questions to explore within the broad categories of the Search for Life and geological processes on the Earth and planets. After choosing a question, students go to their "office" where they are guided through the software by an "investigation cycle diagram." The steps in the research model illustrated for the students are: exploration, background research, data collection, data analysis, and presentation of results. An audiovisual "mentor" provides explanations and suggestions for each step. Following national science standards, the pedagogy is inquiry-based and explores authentic questions to which the answers are not yet known.

One of the research questions within the "Search for Life" topic is the search for earthlike life using natural and infrared color images. Students frist go to the Theater where they hear experts briefly debate over whether or not remote sensing images can be used to detect life. They then examine Landsat images of a heavily forested area in West Virginia and a desert area west of Tucson. Students construct a natural color composite image by combining gray-scale of the scene taken through red, green and blue (RGB) filters. They then create an infrared color composite of the same area using infrared, red, and green (IR, RG) gray-scale images and asked to note how the colors of features like forests, rocks, water, and buildings change between the natural and infrared composites. A "zoom" feature allows students to create color composites of photographs of selected views on the surface within each scene. In this way, students can see that green plants behave in a recognizably different way then other green 
objects in the scenes. Students then look at objects in their school using red, green, and blue filters to drive home how different objects look in different colors. All of this is done in an experiential mode with little explanation. Only after they have made and organized their own observations are they presented with short written summary of the concepts of spectrum and color. Finally, the students go the Control Center to "take" RGB and IR images of their own of Earth and Mars from spacecraft, and surface images of Mars which thy combine using the same format as before into natural and IR composites. They then use the experience they gained from looking at the Earth satellite and surface images to search for life on Mars. Finally, they present their findings to the teacher or to each other in a class-wide "science conference."

Assessments of student learning show that using this approach, students not only learn science content related to the spectrum and color, but also learn how to apply problem-solving skills gained in one situation to another. 